Revue

Revue de l'histoire des religions

de Ihistoire des religions

3 | 2018

Varia

Catherine MAsson, Des laïcs chez les Prêcheurs. De l'ordre de la pénitence aux fraternités laïques, une histoire du tiers-ordre dominicain

Paris, Les Éditions du Cerf, 2016

Sylvie Duval

\title{
OpenEdition
}

Journals

Édition électronique

URL : http://journals.openedition.org/rhr/9101

DOI : 10.4000/rhr.9101

ISSN : 2105-2573

Éditeur

Armand Colin

Édition imprimée

Date de publication : 1 septembre 2018

Pagination : 554-557

ISBN : 978-2-200-93188-9

ISSN : 0035-1423

Référence électronique

Sylvie Duval, « Catherine MAsson, Des laïcs chez les Prêcheurs. De l'ordre de la pénitence aux fraternités laïques, une histoire du tiers-ordre dominicain », Revue de l'histoire des religions [En ligne], 3 | 2018, mis en ligne le 01 septembre 2018, consulté le 15 janvier 2021. URL : http://journals.openedition.org/rhr/9101 ; DOI : https://doi.org/10.4000/rhr.9101

Ce document a été généré automatiquement le 15 janvier 2021.

Tous droits réservés 


\section{Catherine MASSON, Des laïcs chez les Prêcheurs. De l'ordre de la pénitence aux fraternités laïques, une histoire du tiers-ordre dominicain}

Paris, Les Éditions du Cerf, 2016

Sylvie Duval

\section{RÉFÉRENCE}

Catherine MAsson, Des laïcs chez les Prêcheurs. De l'ordre de la pénitence aux fraternités laïques, une histoire du tiers-ordre dominicain, Paris, Les Éditions du Cerf, 2016, 21,5 cm, 294 p., $24 €$, ISBN 978-2-204-11183-6.

Dans son dernier ouvrage, Catherine Masson aborde un sujet ample qui, s'il n'est pas entièrement nouveau, n'a toutefois pas encore fait l'objet d'un traitement systématique, et moins encore d'une inscription dans la longue durée. L'auteure y traite de l'histoire du tiers-ordre dominicain en quatre chapitres chronologiques. Le premier s'attache à décrire les origines médiévales de cette forme de vie religieuse, le deuxième s'applique à en trouver la continuation durant l'époque moderne. Les deux derniers chapitres s'attardent respectivement sur les $\mathrm{XIX}^{\mathrm{e}}$ et $\mathrm{xx}^{\mathrm{e}}$ siècles, période qui vit le tiers-ordre évoluer considérablement, jusqu'à 1985, date de la promulgation de la règle encore en vigueur chez ceux qui sont désormais officiellement appelés «laïcs dominicains ».

C. Masson ne cache pas son manque de familiarité avec l'histoire médiévale et, par conséquent, son renoncement à consulter directement les sources de la période. Pour les périodes suivantes en revanche (et surtout pour la période contemporaine), les références aux sources de première main sont nombreuses, et constitueront pour les chercheurs une mine d'informations précieuse. Cependant le compte rendu de ses 
recherches par l'auteure confine parfois à une énumération sans réelle synthèse. Si l'ouvrage en effet pose de nombreuses questions et aborde des aspects d'un très vif intérêt pour l'histoire religieuse française - nous en nommerons quelques-uns plus bas - certains problèmes fondamentaux ne sont qu'effleurés, remettant en question la cohérence même du sujet.

3 Et avant tout la question de la définition du laïcat : une question à laquelle $\mathrm{C}$. Masson ne répond pas vraiment. De fait, il n'est pas sûr qu'on puisse, avant le $\mathrm{xx}^{\mathrm{e}}$ siècle et Vatican II, définir le laïcat autrement que par la négative. Depuis la réforme grégorienne en effet, ce sont les clercs dont l'Église s'est efforcée de définir le statut, insistant sur la spécificité et l'importance de leur mission, sur leurs droits et leurs privilèges, sur les interdictions afférentes à leur état. Pour les périodes anciennes, les laïcs se définissent donc principalement comme tous les " non clercs ", d'où l'existence, dans la pratique, d'une infinité d'états intermédiaires difficilement classables, à commencer par les femmes, que leur sexe interdit d'être «clercs» mais qui peuvent toutefois être considérées comme des "personnes religieuses». André Vauchez, dans ses importants travaux sur le sujet, a mis en valeur « l'inventivité des laïcs » de la fin du Moyen Âge, mais aussi, en parallèle, «la cléricisation » accélérée de l'Église durant la même période, conduisant au bout du compte à une bien maigre reconnaissance des états religieux non cléricaux par l'institution ecclésiale. Et de fait, la mise en valeur du " laïcat » chez les dominicains se réfère à une époque très récente, ainsi que le précise C. Masson au cours du dernier chapitre, et renvoie à des catégories de pensées issues notamment des travaux d'Yves Congar.

Le critère du « laïcat » n'est donc peut-être pas le plus pertinent pour une analyse du tiers-ordre et des divers mouvements religieux rattachés à l'ordre dominicain sur la longue durée. Il est assez artificiel en effet de faire des "pinzochere" et des « mantellate » des villes médiévales italiennes les précurseurs des laïcs dominicains des sociétés contemporaines, sans parler des tertiaires cloîtrées de l'époque moderne que C. Masson peine à intégrer dans son récit. En outre, l'engagement dans le tiers-ordre peut aussi être le fait de clercs (prêtres chargés de paroisses, prélats) ainsi que le rappelle l'auteure elle-même à diverses reprises. Cette idée de la continuité de l'histoire des tertiaires dominicains à travers les siècles reprend, finalement, dans ses grandes lignes, la «vulgate " interne à l'Ordre remontant aux écrits de Raymond de Capoue (m.1399) et de Thomas de Sienne (m.1431) qui, en vue de la canonisation de la mantellata Catherine de Sienne (obtenue en 1461), avaient associé la promotion du nouveau tiers-ordre dominicain avec le culte de Catherine, devenue ainsi une tertiaire $a$ posteriori.

5 Dans l'optique de longue durée adoptée par l'auteure, il aurait peut-être fallu s'interroger plus en profondeur sur la nature et les raisons de l'engagement particulier de ces fidèles qui, d'une manière ou d'une autre, ont cherché dès l'origine de l'Ordre dominicain à se placer dans sa sphère d'influence. Il faut sans doute comprendre ce type d'engagement, appelé Tiers-Ordre $d u \mathrm{xv}^{\mathrm{e}}$ au $\mathrm{xx}^{\mathrm{e}}$ siècle et présent dans beaucoup d'autres ordres religieux, en particulier chez les franciscains, comme une volonté, de la part de personnes dont la condition particulière les empêche de prendre les vœux (femmes trop pauvres pour entrer au monastère, travailleurs/euses, personnes mariées, chargés de famille, curés, etc.) de se rapprocher malgré tout de la vie régulière dans certains de ses aspects fondamentaux (récitation des heures, support de la communauté, exigence spirituelle individuelle). On peut expliquer de cette manière 
différents phénomènes soulignés par l'auteure, comme la survie de cette "mouvance " de l'Ordre des Prêcheurs à travers des formes extrêmement diverses durant plusieurs siècles, son importance spécifique au $\mathrm{xIX}^{\mathrm{e}}$ siècle au moment même où l'Ordre était moribond (chapitre 3), mais aussi l'apparent « doublon » que constituent, au xxe siècle, le tiers-ordre et l'action catholique (p. 183-204), en réalité nés sur des bases totalement différentes.

remarques qui précèdent ne doivent pas être comprises comme une remise en cause du travail de l'auteure. Bien au contraire, il faut voir ici tout l'intérêt du propos de C. Masson qui, par la mise en évidence des nombreuses formes du tiers-ordre, de ses évolutions, de ses questionnements internes, nous pousse à réviser certaines de nos catégories historiques et, surtout, comme elle le dit elle-même en introduction, à "sortir» d'une histoire interne à l'Ordre dominicain. L'auteure ne s'est, en fin de compte, peut-être pas autorisé toutes les libertés dans ce domaine, mais il ne fait pas de doute que les chercheurs qui ne manqueront pas de prendre son livre comme point de départ pour leur réflexion y trouveront des éléments importants pour leur travail, aussi bien dans les exemples qui y sont développés que dans certaines hypothèses qui y sont proposées.

7 Nous citerons ici quelques-unes des pistes suggérées par cet ouvrage. Et tout d'abord la forte présence des femmes à l'intérieur du tiers-ordre et des mouvances qui lui sont associées, pour toutes les périodes que l'auteure prend en considération (avec une petite exception, peut-être, pour le XIX ${ }^{\mathrm{e}}$ siècle). Que signifie cette surreprésentation ? Il importe ici de s'interroger réciproquement, d'une part, sur la reconnaissance institutionnelle par l'Église de la vocation féminine (puisqu'on trouve parmi les «tertiaires " aussi bien des moniales cloîtrées que des femmes mariées!) et, d'autre part, sur la spécificité « féminine » d'une vocation active et charitable (qui semble être l'un des points communs de ces religieuses à travers les siècles). Par ailleurs, l'auteure souligne à diverses reprises, pour la période contemporaine, les origines sociologiques variées des tertiaires, avec, toutefois, l'exception notable des ouvriers (p.129, p. 173). L'engagement, au xIX ${ }^{\mathrm{e}}$ siècle et au début $\mathrm{du} \mathrm{xx}^{\mathrm{e}}$ siècle, de nombreux artistes dans le tiers-ordre est lui aussi intéressant: l'auteure développe plusieurs exemples significatifs à ce sujet (congrégation Saint-Jean-l'Évangéliste fondée à Paris dès 1839; fraternité du faubourg Saint-Honoré dont furent membres Maurice Denis et Georges Desvallières). Le rapport particulier, enfin, entre Lacordaire et le tiers-ordre fait l'objet d'une réflexion poussée sur les liens unissant l'Ordre dominicain aux laïcs et, plus largement, à la société française alors en pleine évolution, notamment du point de vue du rapport à la vocation religieuse et à la présence des religieux (et plus largement de la religion) dans l'espace public. L'ouvrage de Catherine Masson contribue ainsi avec profit au renouvellement des études sur les différentes formes d'engagement religieux "dans le monde " au cours des siècles, et permettra sans nul doute l'émergence de questionnements nouveaux à ce propos. 


\section{AUTEURS}

\section{SYLVIE DUVAL}

Fondation Thiers / CIHAM, Lyon. 